\title{
Screening of circular RNAs and validation of circANKRD36 associated with inflammation in patients with type 2 diabetes mellitus
}

\author{
YUAN FANG ${ }^{1}$, XIAOXIA WANG ${ }^{2}$, WENQING LI ${ }^{1}$, JINGLI HAN $^{1}$, JUNHUA JIN ${ }^{1}$, FEI SU $^{3}$, \\ JUNHUA ZHANG $^{1}$, WEI HUANG ${ }^{1}$, FEI XIAO ${ }^{1,3}$, QI PAN ${ }^{2}$ and LIHUI ZOU ${ }^{1}$ \\ ${ }^{1}$ The MOH Key Laboratory of Geriatrics; Departments of ${ }^{2}$ Endocrinology and ${ }^{3}$ Pathology, \\ Beijing Hospital, National Center of Gerontology, Beijing 100730, P.R. China
}

Received February 7, 2018; Accepted July 6, 2018

DOI: $10.3892 / \mathrm{ijmm} .2018 .3783$

\begin{abstract}
Circular RNAs (circRNAs) are an abundant class of endogenous non-coding RNAs and are associated with numerous diseases, including cancer, cardiovascular diseases, and type 2 diabetes mellitus (T2DM). However, the association between circRNAs and inflammation or inflammatory cytokines in patients with T2DM remains to be fully elucidated. The purpose of the present study was to investigate the expression profiles of circRNAs in peripheral leucocytes of patients with T2DM and their association with inflammatory cytokines. Peripheral blood from patients with T2DM $(n=43)$ and healthy individuals $(\mathrm{n}=45)$ were collected for RNA sequencing and later verification. Reverse transcription-polymerase chain reaction (RT-PCR) and reverse transcription-quantitative polymerase chain reaction (RT-qPCR) analyses were used to detect the expression levels of circRNAs. The expression of inflammatory factors, including interleukin (IL)-1, (IL)-6, and tumor necrosis factor (TNF)- $\alpha$ were measured via enzyme-linked immunosorbent assay. Furthermore, the mRNA expression level of ankyrin repeat domain 36 (ANKRD36), a protein located at 2q11.2 that interacts with the GAPDH gene, was measured using RT-qPCR analysis. The circRNA/microRNA (miRNA) interaction was predicted
\end{abstract}

Correspondence to: Professor Lihui Zou, The MOH Key Laboratory of Geriatrics, Beijing Hospital, National Center of Gerontology, 1 Dahua Road, Dongcheng, Beijing 100730, P.R. China E-mail: zoulihui4371@bjhmoh.cn

Abbreviations: circRNAs, circular RNAs; T2DM, type 2 diabetes mellitus; GLU, glucose; GHb, glycosylated hemoglobin; RNAseq, RNA sequencing; RT-PCR, reverse transcription-polymerase chain reaction; RT-qPCR, reverse transcription-quantitative polymerase chain reaction; ELISA, enzyme-linked immunosorbent assay; miRNA, microRNA

Key words: circular RNAs, type 2 diabetes mellitus, inflammation, biomarker, RNA sequencing using RegRNA and mirPath software. In total, 220 circRNAs were found to be differentially expressed between patients with T2DM and healthy individuals, of which 107 were upregulated and 113 were downregulated. Among the nine selected circRNAs, circANKRD36 was significantly upregulated in patients with T2DM compared with control subjects $(\mathrm{P}=0.02)$. The expression level of circANKRD36 was positively correlated with glucose and glycosylated hemoglobin $(\mathrm{r}=0.3250, \mathrm{P}=0.0047$ and $\mathrm{r}=0.3171, \mathrm{P}=0.0056$, respectively). The expression level of IL-6 was significantly different between the T2DM group and control group $(\mathrm{P}=0.028)$ and was positively correlated with circANKRD36. The difference of circANKRD36 host gene expression between patients with T2DM and healthy controls was significant $(\mathrm{P}=0.04)$. Taken together, circANKRD36 may be involved in T2DM and inflammation-associated pathways via interaction with miRNAs, including hsa-miR-3614-3p, hsa-miR-498, and hsa-miR-501-5p. The expression of circANKRD36 was upregulated in peripheral blood leucocytes and was correlated with chronic inflammation in T2DM. Therefore, circANKRD36 can be used as a potential biomarker for screening chronic inflammation in patients with T2DM.

\section{Introduction}

Type 2 diabetes mellitus (T2DM) is a chronic metabolic disorder characterized by hyperglycemia, insulin resistance and chronic inflammation (1,2). According to the International Diabetes Federation Diabetes Atlas (3), almost 400,000,000 individuals suffer from T2DM globally, and $>10,000,000$ are diagnosed with diabetes every year. The prevalence of T2DM in China has increased substantially over recent years, with $>100,000,000$ individuals affected by the disease at present (4). Hyperglycemia, insulin resistance and chronic inflammation are common in patients with T2DM. Chronic, low-grade inflammation can lead to insulin resistance, impaired glucose tolerance and finally diabetes. Hyperglycemia and overnutrition stimulate the production of inflammatory cytokines, which in turn, aggravate glucotoxicity and insulin resistance, leading to numerous life-threatening complications $(1,5)$. Therefore, early diagnosis and intervention of chronic inflammation in 
patients with T2DM are urgently required. However, no diagnostic biomarker for inflammation in patients with T2DM is available. To improve this situation, a specific, sensitive and convenient biomarker for inflammation in T2DM is urgently required.

Circular RNAs (circRNAs), have been considered as the 'dark matter' of the genome due to their substantial diversity and unclear function. However, with the development of next generation sequencing, circRNAs have been revealed to be more important than previously considered. circRNAs may function as 'microRNA (miRNA) sponges', which regulate cellular function at the post-transcription stage $(6,7)$. Another important function of circRNAs is that they are able to regulate host gene expression through cis-acting $(8,9)$. In addition, as circRNAs are derived from back splicing, which enables them to form continuous loops without free $3^{\prime}$ and 5 ' ends, they are more resistant to RNA exonuclease and are more stable compared with corresponding linear isoforms in cells (10). circRNAs also exhibit cell type-specific and tissue type-specific expression (11). Due to these features, circRNAs are expected to become diagnostic biomarkers and treatment targets in human diseases.

circRNAs have been revealed to be associated with T2DM. For example, hsa_circ0054633 in the peripheral blood may be used as a diagnostic biomarker of pre-diabetes and T2DM; another circRNA, Cdrlas, regulates insulin transcription and secretion in islet cells via miRNA (miR)-7 and its targets $(12,13)$. However, the global circRNA expression profile in the peripheral white blood cells of patients with T2DM remains to be fully elucidated. In the present study, RNA sequencing (RNAseq) was used to examine the expression of circRNAs, and their association with chronic inflammation in T2DM was investigated. In total, 220 differentially expressed circRNAs were identified in patients with T2DM compared with healthy subjects. Of these, nine circRNAs were selected and measured by reverse transcription-polymerase chain reaction (RT-PCR) and reverse transcription-quantitative polymerase chain reaction (RT-qPCR) analyses. Subsequent investigations focused on circRNA ankyrin repeat domain 36 (circANKRD36), one of the circRNAs with the most markedly increased expression. RT-qPCR analysis was used to validate its expression level and its correlation with glucose (GLU) and glycosylated hemoglobin (GHb). An enzyme-linked immunosorbent assay (ELISA) was performed to measure the expression levels of inflammatory factors, and interleukin (IL-6) was promoted in theT2DM group. The circANKRD36 host gene was upregulated in patients with T2DM, and the interactions between circANKRD36 and miRNAs were predicted using RegRNA and mirPath software.

\section{Materials and methods}

Study population and T2DM definition. In the present study, a total of 88 individuals were included, including 43 patients with T2DM and 45 age- and sex-matched control individuals. The patients enrolled were among the outpatients and inpatients of the Endocrinology Department and the Medical Check-up Center of Beijing Hospital (Beijing, China) between October 2016 and May 2017. Participants were excluded if they had: i) Any other clinically systemic acute or chronic inflammation disease/s; ii) autoimmune disease; iii) any endocrine disease other than T2DM; iv) cancer; or v) chronic hepatic or renal failure. The study was approved by the Ethics Committee of Beijing Hospital (no. 2015BJYYEC-066-02). The participants provided written informed consent to participate in the study.

T2DM was diagnosed according to the World Health Organization criteria: i) Fasting glucose level $>7 \mathrm{mmol} / \mathrm{l}$; or ii) the 2-h oral glucose tolerance test showing a glucose level of $\geq 11.1 \mathrm{mmol} / \mathrm{l}$; or iii) hemoglobin A1c $\geq 6.5 \%$, or iv) the subject has a clinical diagnosis of the disease. The basic information of the patients with T2DM and control individuals are listed in Tables I and II.

Blood sample collection and RNAseq. Following overnight fasting, $2 \mathrm{ml}$ of intravenous blood was drawn into ethylene diamine tetra-acetic acid anticoagulant vacutainers. The whole blood cell specimens of five patients with T2DM and five control subjects were randomly selected for RNAseq. Total RNAs were extracted with TRIzol (Invitrogen; Thermo Fisher Scientific, Inc., Waltham, MA, USA), and rRNAs were deleted using Ribo-Zero Gold kits (Illumina, Inc., San Diego, CA, USA). The total RNAs were then digested by RNase R to enrich circRNAs. The cDNAs were amplified with random primers and reverse transcriptase prior to RNAseq using the high-seq 4000 Illumina platform. The whole detection was performed under the guidance of the experiment workflow. Other specimens were centrifuged at $3,000 \mathrm{x}$ g at $4^{\circ} \mathrm{C}$ for $5 \mathrm{~min}$ to separate plasma and blood cells, and stored separately in a $-80^{\circ} \mathrm{C}$ refrigerator for later usage.

RNA extraction and RT. Total RNAs in leucocytes and plasma were extracted with TRIzol and TRIzol LS (Invitogen; Thermo Fisher Scientific, Inc.), respectively by following the manufacturer's protocol. The purity and concentration of the RNA were measured using a Nano Drop 2000 instrument (Thermo Fisher Scientific, Inc.). The average A260/A280 was $\sim 1.8-2.0$. First-strand cDNA was amplified with random primers using the GoScript Reverse Transcription system (Promega Corporation, Madison, WI, USA) in accordance with the manufacturer's protocol. Briefly, a total of $2 \mu \mathrm{g}$ RNA and $2 \mu \mathrm{l}$ random primers were added to prepare the annealing mix. The mixture was incubated at $70^{\circ} \mathrm{C}$ for $5 \mathrm{~min}$, and then immediately chilled on ice for $5 \mathrm{~min}$. The annealing mix were stored on ice until the reverse transcription reaction mix was added ( $4 \mu \mathrm{l}$ GoScript $5 \mathrm{X}$ reaction buffer, $2 \mu \mathrm{l} \mathrm{MgCl}_{2}, 1 \mu \mathrm{l}$ dNTP and $1 \mu \mathrm{l}$ GoScript reverse transcriptase). The mixture was incubated at $25^{\circ} \mathrm{C}$ for $5 \mathrm{~min}, 42^{\circ} \mathrm{C}$ for $1 \mathrm{~h}$, and then $70^{\circ} \mathrm{C}$ for $15 \mathrm{~min}$ to inactivate the reverse transcriptase.

RT-PCR and RT-qPCR analyses. Divergent primers and convergent primers were designed by Primer3 (http://www-genome. ut.ee/) and synthesized by Tsingke Technology (Beijing, China). The primer sequences are listed in the Table III. PCR was performed using Golden Mix (Tsingke Technology) and $2 \%$ agarose gel electrophoresis was used to confirm the PCR products. According to the manufacturer's protocol, $12.5 \mu 12 \mathrm{X}$ Super PCR Mix, $1 \mu \mathrm{l} 10 \mu \mathrm{M}$ forward primers, $1 \mu \mathrm{l} 10 \mu \mathrm{M}$ reverse primers, $1 \mu \mathrm{l}$ cDNA, $5 \mu \mathrm{l} 5 \mathrm{X}$ buffer and $4.5 \mu \mathrm{l}$ nuclease-free water were mixed together. The thermal cycling conditions were 
Table I. Basic information of control and patient samples used for RNAseq.

\begin{tabular}{lccc}
\hline Factor & Control $(\mathrm{n}=5)$ & T2DM $(\mathrm{n}=5)$ & P-value \\
\hline Age & $65.2 \pm 13.23$ & $66.2 \pm 5.49$ & 0.894057 \\
Sex & & & - \\
Male & 2 & 2 & \\
Female & 3 & 3 & 0.000728 \\
GLU & $5.16 \pm 0.46$ & $10.7 \pm 1.39$ & 0.005336 \\
GHb & $5.56 \pm 0.24$ & $9.08 \pm 1.33$ & \\
\hline
\end{tabular}

Data are presented as the mean \pm standard deviation. T2DM, type II diabetes mellitus; GLU, glucose; GHb, glycosylated hemoglobin.

Table II. Basic information of control and patient samples used for verification.

\begin{tabular}{lccc}
\hline Factor & Control $(\mathrm{n}=40)$ & T2DM $(\mathrm{n}=38)$ & P-value \\
\hline Age & $61.15 \pm 11.72$ & $62.53 \pm 12.45$ & 0.621636 \\
Sex & & & 0.364505 \\
Male & 21 & 16 & \\
Female & 19 & 22 & \\
GLU & $5.14 \pm 0.41$ & $10.00 \pm 2.77$ & $5.805904 \times 10^{-13}$ \\
GHb & $5.37 \pm 0.31$ & $8.88 \pm 1.41$ & $7.107031 \times 10^{-18}$ \\
\hline
\end{tabular}

Data are presented as the mean \pm standard deviation. T2DM, type II diabetes mellitus; GLU, glucose; GHb, glycosylated hemoglobin.

as follows: Initial denaturation at $98^{\circ} \mathrm{C}$ for $2 \mathrm{~min}, 30$ cycles of denaturation at $98^{\circ} \mathrm{C}$ for $10 \mathrm{sec}$, annealing at $56^{\circ} \mathrm{C}$ for $10 \mathrm{sec}$, extension at $72^{\circ} \mathrm{C}$ for $15 \mathrm{sec}$, followed by final extension at $72^{\circ} \mathrm{C}$ for 2 min. The RT-qPCR was performed using SYBR-Green Premix Ex Taq (Takara Biotechnology Co., Ltd., Dalian, China) and monitored using theiQ5 real-time PCR system (Bio-Rad Laboratories, Inc., Hercules, CA, USA) in accordance with the protocol. The thermal cycling conditions were as follows: Initial denaturation at $95^{\circ} \mathrm{C}$ for $30 \mathrm{sec}, 40$ cycles of denaturation at $95^{\circ} \mathrm{C}$ for $5 \mathrm{sec}$, annealing at $60^{\circ} \mathrm{C}$ for $30 \mathrm{sec}$, followed by 81 cycles of dissociation at $55^{\circ} \mathrm{C}$ for $30 \mathrm{sec}$. The relative expression was analyzed using the $2^{-\Delta \Delta \mathrm{Cq}}$ method (14).

ELISA. The plasma samples were stored in a $-80^{\circ} \mathrm{C}$ refrigerator and brought to room temperature prior to usage. The levels of IL-1, IL-6 and TNF- $\alpha$ antigen were measured using a Total Antigen Assay ELISA kit (Cloud-Clone Corporation, Wuhan, China). The standard, blank and sample dilutions were added into the appropriate wells and incubated for $1 \mathrm{~h}$ at room temperature. Subsequently, the detection reagent, substrate solution and stop solution were added in turn, according to the manufacturers' protocol. Measurements were performed at $450 \mathrm{~nm}$ immediately.

Target prediction and functional analysis. Those miRNAs able to interact with circRNAs were predicted using RegRNA
2.0 (http://www.regrna.mbc.nctu.edu.tw/) (15). A network between the predicted miRNAs and their downstream pathways was constructed using miRPathv.3 (http://vm.okeanos. grnet.gr/) software according to the common targets (16).

Statistical analysis. All statistical analysis in the present study was performed using the Statistical Product and Service Solutions (SPSS) 20.0 software package (IBM SPSS, Armonk, NY, USA) and GraphPad Prism 6.0 (GraphPad Software, Inc., La Jolla, CA, USA). Data were presented as the mean \pm standard deviation of three independent experiments performed in triplicate. Independent unpaired Student's t-test, one-way analysis of variance with a Student-Newman-Keuls post-hoc test and Pearson's correlation analysis were used, as appropriate. $\mathrm{P} \leq 0.05$ was considered to indicate a statistically significant difference.

\section{Results}

Profiles of circRNA expression in the peripheral blood cells of patients with T2DM. The RNAseq analysis revealed the circRNA expression profiles in the peripheral white blood cells of five paired patients with T2DM and healthy individuals. The circRNA expression pattern was found to be different between the T2DM group and control group (Fig. 1A). In total, 3,048 circRNAs were detected in both T2DM samples and control samples. Differential expression was detected in 220 circRNAs, with the level of change $>1.5$-fold. Among these, 107 were upregulated and 113 were downregulated (Fig. 1B and C). As upregulated circRNAs are more applicable as biomarkers than downregulated circRNAs, nine upregulated circRNAs were selected for further analysis.

Validation of differentially-expressed circRNAs by RT-qPCR and RT-PCR analyses. To validate the RNAseq results, RT-qPCR and RT-PCR analyses were performed to analyze the differential expression. According to the RT-qPCR results, the upregulation of three circRNAs (circANKRD36, hsa_circ_0008922 and hsa_circ_0009035) was successfully detected in the T2DM samples (Fig. 2A). Among these, the expression level of circANKRD36 was the most markedly increased. As circANKRD36 is a newly identified circRNA derived from $A N K R D 36$, it was named after its host gene. Hsa_circ_0008922 and hsa_circ_0009035 have already been reported by others $(10,17)$. RT-PCR analysis for circANKRD36 was performed, and the back-splicing junction was validated by sequencing the product (Fig. 2B and C), which was consistent with that from the RNAseq data. Therefore, circANKRD36 was selected for further analysis.

Validation of circANKRD36 in larger clinical samples. To further determine the association between circANKRD36 and T2DM, the present study first detected the expression of circANKRD36 in additional patients by RT-PCR analysis, using $\beta$-actin as a control (Fig. 3A). RT-qPCR analysis was then performed to measure the level of circANKRD36 in a cohort containing 78 patients, comprising 38 patients with T2DM and 40 healthy individuals. As shown in Fig. 3B, the expression of circANKRD36 was significantly higher in the T2DM group than in the control group $(0.2458 \pm 0.021$, vs. 
Table III. Primer list.

Gene

circANKRD36

hsa_circ_0008922

hsa_circ_0009035

ANKRD36

$\beta$-actin
Forward primer $\left(5^{\prime}-3^{\prime}\right)$

Reverse primer (5'-3')

circ, circular RNA; ANKRD36, ankyrin repeat domain 36.

$0.1831 \pm 0.017 ; \mathrm{P}=0.02)$. Pearson's correlation test was performed to evaluate the association between circANKRD36 and levels of GLU and GHb. As shown in Fig. 3C and D, circANKRD36 was positively correlated with GLU $(r=0.3250, \mathrm{P}=0.0047)$ and $\mathrm{GHb}(\mathrm{r}=0.3171, \mathrm{P}=0.0056)$. However, circANKRD36 was not detected in the peripheral blood plasma samples from patients with T2DM (Fig. 3E).

ELISA. The expression levels of inflammatory factors (IL-1, IL-6, and TNF- $\alpha$ ) in the peripheral blood plasma were analyzed by ELISA. As shown in Table IV, the expression levels of IL-6 and TNF- $\alpha$ were elevated in patients with T2DM, compared with those in control subjects $(13.202 \pm 9.937$, vs. $6.151 \pm 3.787$, and $16.158 \pm 5.964$, vs. 13.165 \pm 2.69 , respectively). However, only the increase of IL-6 was statistically significant $(\mathrm{P}=0.028)$.

Pearson's correlation test was used to examine the correlation between circANKRD36 and inflammatory factors. As shown in Fig. 4A-C, IL-6 was positively correlated with circANKRD36 $(\mathrm{r}=0.393, \mathrm{P}=0.031)$. Although the elevation of $\mathrm{TNF}-\alpha$ was not statistically significant $(\mathrm{P}=0.080)$, its elevation was positively correlated with circANKRD36 $(r=0.390, \mathrm{P}=0.033)$.

Cis-regulation of the circANKRD36 host gene. The mRNA expression level of ANKRD36 was measured by RT-qPCR analysis in 15 paired patients with T2DM and control individuals. In the T2DM group, circANKRD36 host gene expression was higher than that in the control group $(0.006454 \pm 0.003682$, vs. $0.003698 \pm 0.00138, \mathrm{P}=0.04$; Fig. $5 \mathrm{~A})$. The location and associated genes of ANKRD36 were also detected (Fig. 5B and C).

Prediction of the circRNA/miRNA interaction. To further investigate the functions of circANKRD36, the present study investigated the potential miRNAs binding with the circRNA. The binding sites were predicted using RegRNA, based on the foundation of seed sequence matching. circANKRD36 harboredhsa-miR-3614-3p, hsa-miR-498, hsa-miR-501-5p, hsa-miR-876-3p, hsa-miR-3617, hsa-miR-4251 and hsa-miR-2355-3p (Table V). mirPath software analysis determined statistically significant miRNA downstream pathways $(\mathrm{P}<0.05$; Fig. 6).

\section{Discussion}

The high prevalence of T2DM and its complications severely threaten human health. Chronic inflammation exists in almost every patient with T2DM and is closely associated with the
Table IV. Enzyme-linked immunosorbent assay results.

\begin{tabular}{lccc}
\hline Factor & IL-1 & IL-6 & TNF- $\alpha$ \\
\hline T2DM & $13.227 \pm 1.138$ & $13.202 \pm 9.937$ & $16.158 \pm 5.964$ \\
Control & $13.032 \pm 1.463$ & $6.151 \pm 3.787$ & $13.165 \pm 2.692$ \\
P-value & 0.695 & $0.028^{\mathrm{a}}$ & 0.080 \\
\hline
\end{tabular}

Data are presented as the mean \pm standard deviation. ${ }^{\text {a Statistically }}$ significant $(\mathrm{P}<0.05)$. T2DM, type II diabetes mellitus; IL, interleukin; TNF- $\alpha$, tumor necrosis factor- $\alpha$.

pathophysiological alterations occurring during the pathogenesis of T2DM (18). Multiple investigations have revealed that anti-inflammatory strategies may correct the disordered metabolism in patients with T2DM $(1,18)$. However, the molecular mechanism underlying inflammation in patients with T2DM remains to be fully elucidated.

circRNAs have been found to be pervasively transcribed in the genome, and associated with human disease. Certain circRNAs are involved in the occurrence and development of T2DM, whereas others are associated with T2DM and may be used as biomarkers $(12,13)$. However, the association between circRNAs and inflammation, particularly chronic, low-grade inflammation, in patients with T2DM has not been investigated previously.

In the present study, circRNA expression profiles in peripheral white blood cells from patients with T2DM were investigated using RNAseq. This identified $>200$ circRNAs as being differently expressed between patients with T2DM and healthy individuals. The differential circRNAs were further analyzed through Gene Ontology (GO) analysis. Metabolic process and immune system process were among the top altered GO processes in the biological process category, indicating the possibility that differential circRNAs from peripheral leukocytes were, in general, associated with chronic inflammation in patients with T2DM.

Previous studies have revealed that circRNAs in the cytoplasm are more stable than corresponding linear isoforms (10). Furthermore, the designation of divergent primers guarantees the convenient and specific amplification of target circRNAs, making circRNAs appealing biomarkers for human diseases. In the present study, the aberrant expression of circANKRD36 was verified by RT-PCR and RT-qPCR analyses in peripheral 
A

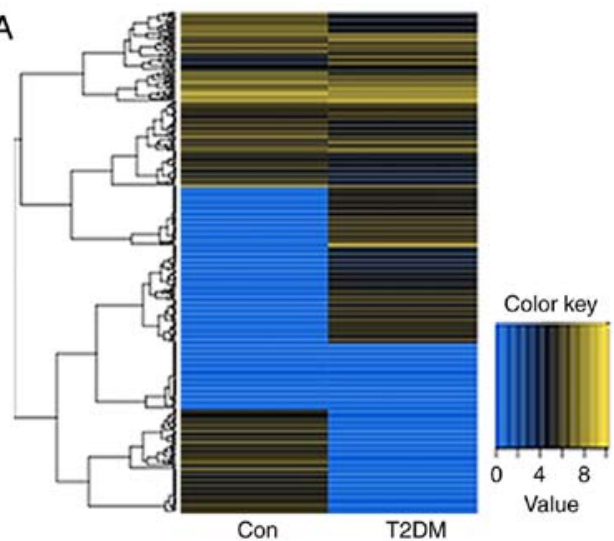

C 250

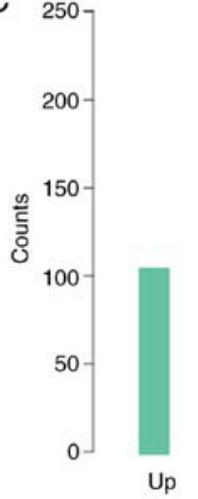

B

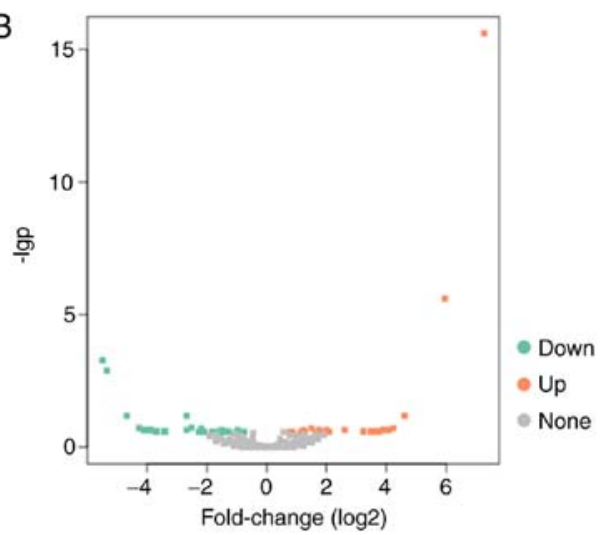

Figure 1. Overview of circRNA differential expression. (A) Hierarchical cluster analysis (heat map) for differentially expressed circRNAs between controls and patients with T2DM. Yellow and blue denote high and low expression, respectively. (B) Volcano plots for differentially expressed circRNAs in leucocytes from control and T2DM groups. (C) Numbers of differential circRNAs. T2DM, type II diabetes mellitus; Con, control; circRNAs, circular RNAs; Up, upregulated; Down, downregulated.
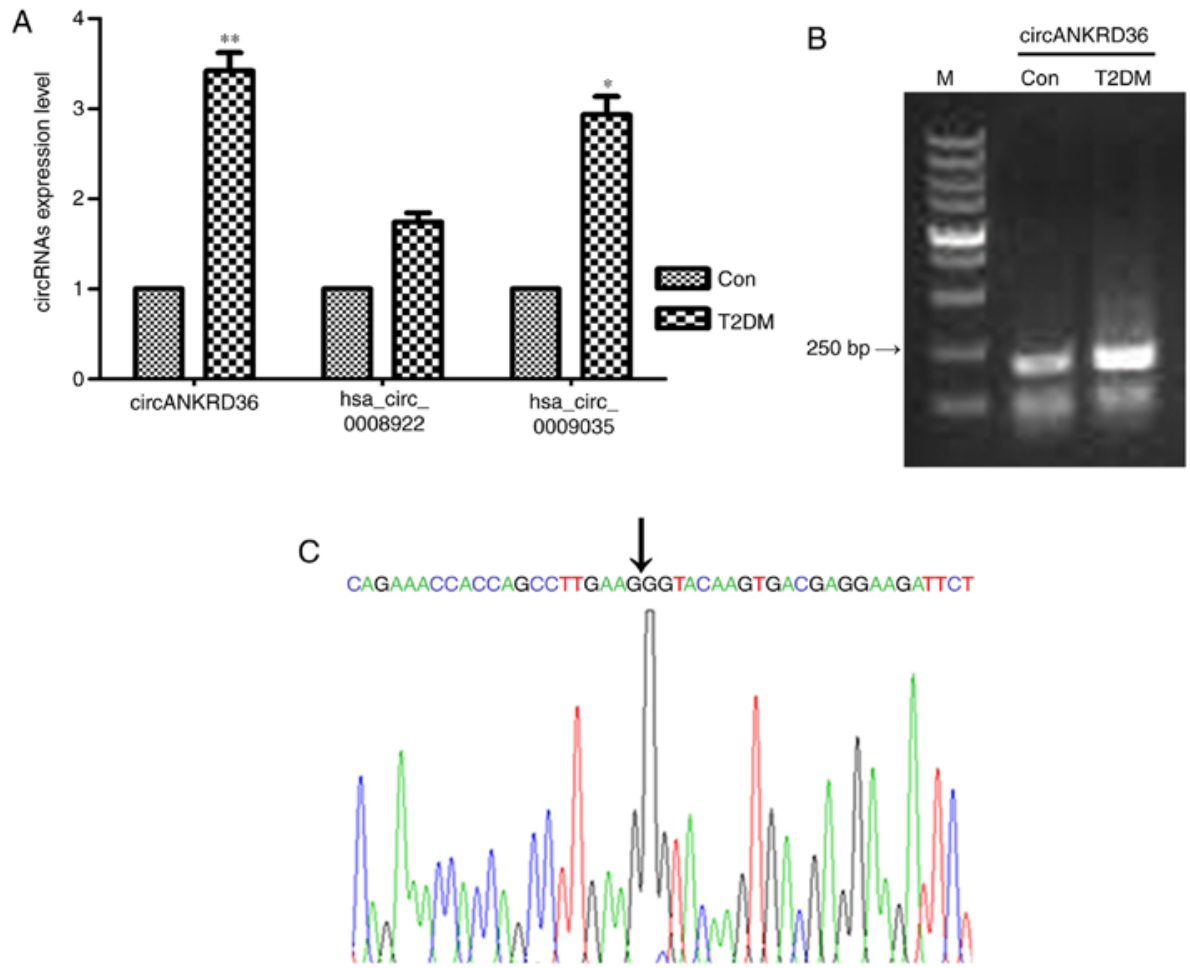

Figure 2. Expression and selection of circANKRD36. (A) Relative expression level of three selected circRNAs in the control group and T2DM group. RT-quantitativePCR was used to determine the expression level. Data are presented as the mean \pm standard deviation. (B) Electrophoresis result of RT-PCR products. Lane M denotes the DL5000 marker. (C) Validation of the circANKRD36 splicing junction site through sequencing of the RT-PCR product. The arrow indicates the splicing junction site. RT-PCR, reverse transcription-polymerase chain reaction. T2DM, type II diabetes mellitus; Con, control; circRNA, circular RNA; ANKRD36, ankyrin repeat domain 36. 

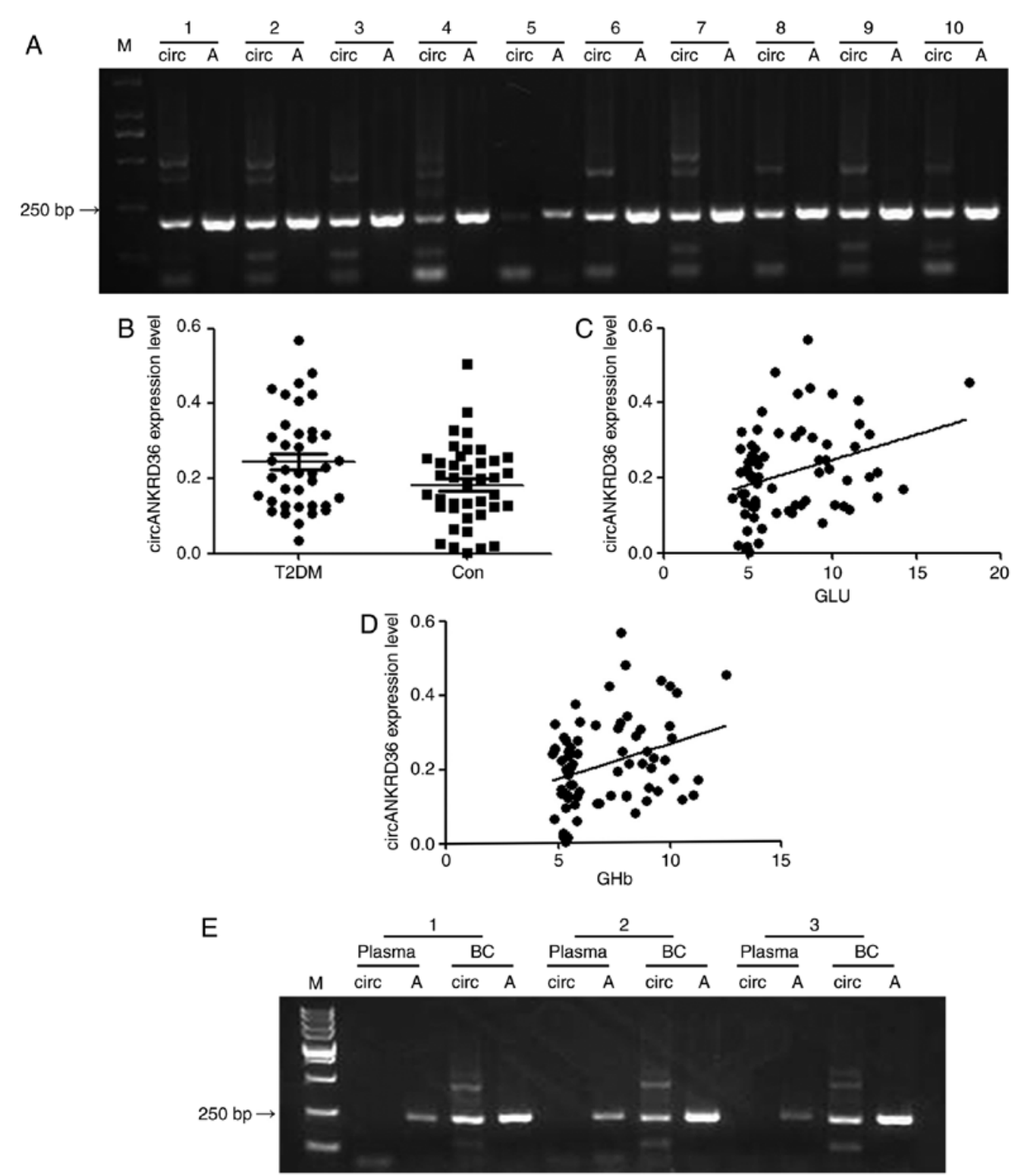

Figure 3. Expression of circANKRD36 is upregulated in patients with T2DM. (A) Electrophoresis result of RT-PCR products from circANKRD36 in 10 patients with T2DM. (B) Increased expression of circANKRD36 in a larger cohort. The expression level was determined by RT-qPCR analysis (n=78, P<0.05). (C) Pearson's coefficient correlation between circANKRD36 and GLU ( $r=0.3250, P=0.0047)$. (D) Pearson's coefficient correlation between circANKRD36 and $\mathrm{GHb}(\mathrm{r}=0.3171, \mathrm{P}=0.0056)$. (E) Electrophoresis result of circANKRD36 and $\beta$-actin. The cDNAs of three patients' peripheral blood plasma were used. T2DM, type II diabetes mellitus; Con, control; circANKRD36, circular RNA ankyrin repeat domain 36; circ, circANKRD36; A, $\beta$-actin control; M, marker; GLU, glucose; GHb, glycosylated hemoglobin; BC, peripheral blood cells.

blood cells. The expression level of circANKRD36 was positively correlated with clinical indicators GLU and GHb when the sample data of patients with T2DM and healthy individuals were combined (Fig. 3C and D), suggesting a potential role for circANKRD36 as a noninvasive hematological biomarker. However, the positive correlation was not be observed when the sample data of patients with T2DM and controls were analyzed separately (data not shown). The reason for this may be the small sample size, therefore, the results of the present study require further verification in larger cohorts. In addition, the increased expression of circANKRD36 was not be detected in peripheral plasma.

In order to investigate the inflammatory state in patients with T2DM, ELISA was performed using blood plasma and the expression levels of IL-1, IL- 6 and TNF- $\alpha$ were measured.
These three inflammatory factors were selected as previous reports have indicated that TNF- $\alpha$ is important in insulin resistance and diabetes $(19,20)$; elevated levels of IL-6 are a predictor for the development of T2DM (21); and IL-1 is associated with islet cell function, with anti-IL-1 therapy having a mild hypoglycemic effect in patients with T2DM $(22,23)$. In the present study, IL- 6 and TNF- $\alpha$ exhibited a positive correlation with circANKRD36, suggesting an association between circANKRD36 and inflammation in T2DM. However, similar trends were not observed for IL-1, and the increase of TNF- $\alpha$ was not significant. In future investigations, additional circRNAs as biomarkers and more participants are to be included in further investigations.

The host gene of circANKRD36, ANKRD36, was upregulated in the T2DM group. Lines of evidence suggest that 
Table V. Top seven target microRNAs.

\begin{tabular}{|c|c|c|c|}
\hline Name & 2D structure & Target region & Score \\
\hline \multirow[t]{2}{*}{ hsa-miR-3614-3p } & Query: 3' uuUUGUGGUUCUAGACUUCCGAu 5' & & \\
\hline & Ref: 5' aaACCACCAGGCT-TGAAGGCTt 3' & 85-106 & 172.00 \\
\hline \multirow[t]{2}{*}{ hsa-miR-498 } & Query: 3' cuUUUUGCGGGGGACCGAACUUu 5' & & \\
\hline & Ref: 5' ctGAGAAACCACCAGGCTTGAAg 3' & $80-102$ & 165.00 \\
\hline \multirow[t]{2}{*}{ hsa-miR-501-5p } & Query: 3' agaGUGGGUC-CC-UGUUUCCUAa 5' & & \\
\hline & Ref: 5' ataTAGCCAGAGGAAAAAAGGATg 3' & $134-157$ & 159.00 \\
\hline \multirow[t]{2}{*}{ hsa-miR-876-3p } & Query: 3' acUUAAUGAA-AC-AUUUGGUGGu 5' & & \\
\hline & Ref: 5' acAGTGTCTTCTGAGAAACCACCa 3' & $70-93$ & 156.00 \\
\hline \multirow[t]{2}{*}{ hsa-miR-3617 } & Query: 3' gggUAGAACGUUGAUACAGAAa 5' & & \\
\hline & Ref: 5' aaaATCTAG-GACAGTGTCTTc 3' & $60-80$ & 146.00 \\
\hline \multirow[t]{2}{*}{ hsa-miR-4251 } & Query: 3' aaccgggAAAAGAGUCc 5' & & \\
\hline & Ref: 5' gaaagtgTCTTCTCAGa $3{ }^{\prime}$ & $273-289$ & 142.00 \\
\hline \multirow[t]{2}{*}{ hsa-miR-2355-3p } & Query: 3' uagaggUUUGUCGUUCCUGUUa 5' & & \\
\hline & Ref: 5' atggagAAAAATCTAGGACAGt 3' & $53-74$ & 140.00 \\
\hline
\end{tabular}

miR, microRNA.
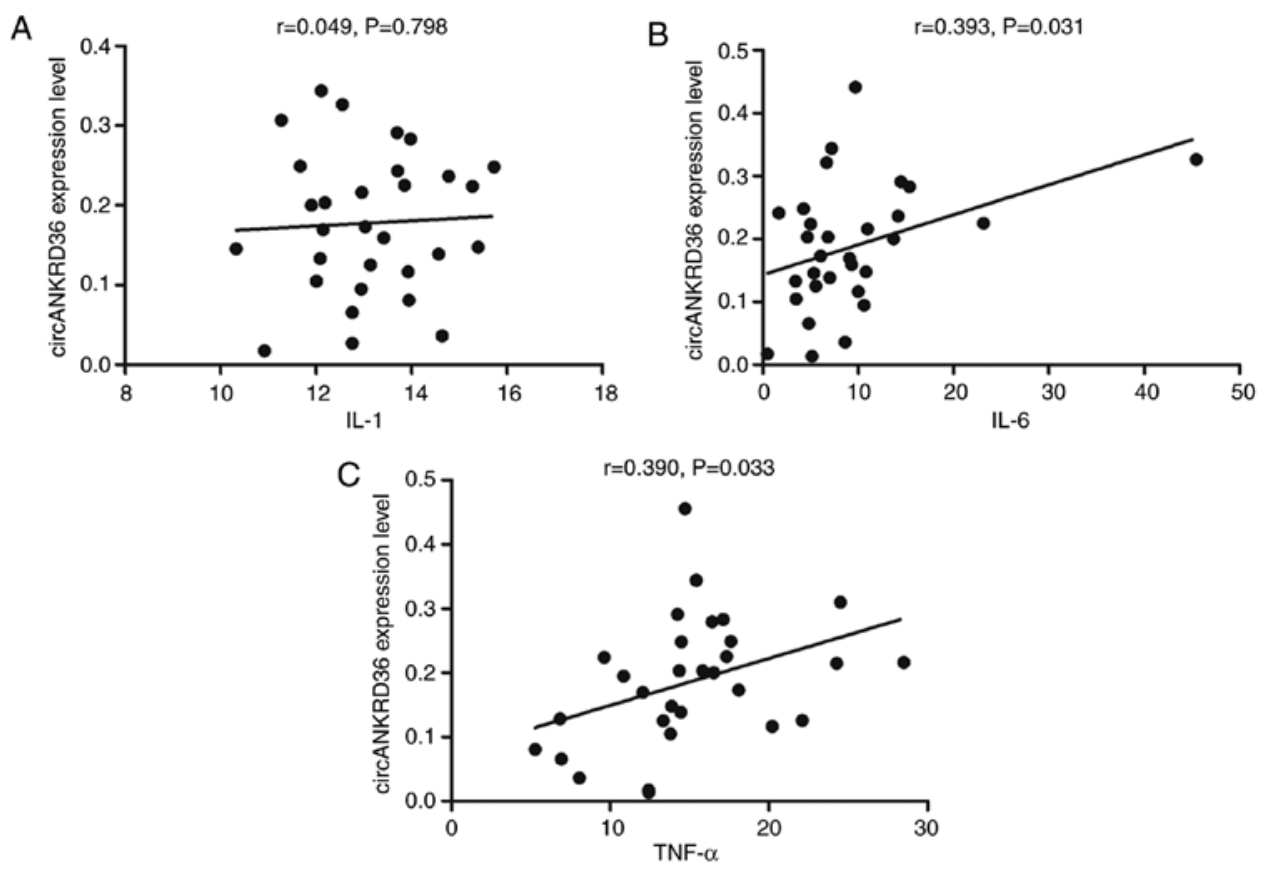

Figure 4. Correlation between the expression levels of circANKRD36 and inflammatory factors. (A) IL-1; (B) IL-6; (C) TNF- $\alpha$. circANKRD36, circular RNA ankyrin repeat domain 36 ; IL-1, interleukin 1; IL-6, interleukin 6 ; TNF- $\alpha$, tumor necrosis factor- $\alpha$.

circRNAs can positively regulate their parent genes at the transcriptional level and post-transcriptional level $(8,9,24)$. The upregulation of ANKRD36 in the present study may be a result of circANKRD36 elevation. ANKRD36 is located close the middle of chromosome 2 at a position designated q11.2 (chr2:97862453-97867967; Fig. 5B), and it can interact with the GAPDH gene (Fig. 5C). The product of GAPDH is a vital enzyme in carbohydrate metabolism. It has been demonstrated that the interaction between glycolytic protein GAPDH and inflammatory protein Small ubiquitin-like modifier 4 can induce insulin resistance in diabetic and obese individuals (25). Therefore, circANKRD36 may be involved in T2DM metabolic disorder by affecting the host gene $A N K R D 36$ and associated gene $G A P D H$.

Bioinformatics software programs (RegRNA and mirPath) have been used to determine circRNA/miRNA interactions and related downstream pathways. Previous studies have reported that circRNAs can function as miRNA sponges $(6,7)$. By sharing one or more microRNA response elements, circRNAs can bind to miRNAs, which in turn adjust the expression of mRNAs. circANKRD36 can interact with seven miRNAs (Table V). Of these, hsa-miR-3617 and hsa-miR-3614-3p are 


\section{A}

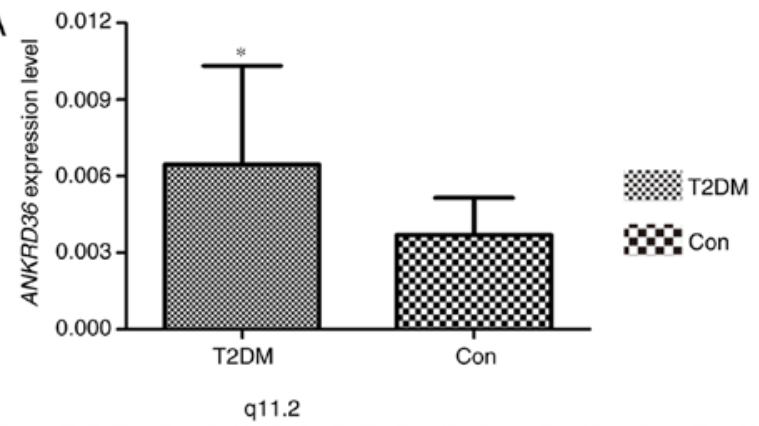

B

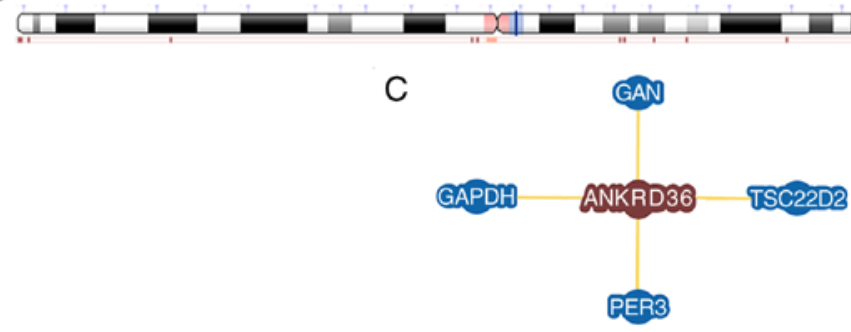

Figure 5. Host gene of circular RNA ANKRD36. (A) Reverse transcription-quantitative polymerase chain reactiondetermination of the expression levels of $A N K R D 36$ in the T2DM group and control group. The graph shows the ratio compared with the housekeeping gene $\beta$-actin. Data are presented as the mean \pm standard deviation. (B) Location of ANKRD36. (C) Associated genes of ANKRD36. T2DM, type 2 diabetes mellitus; Con, control; ANKRD36, ankyrin repeat domain 36 .

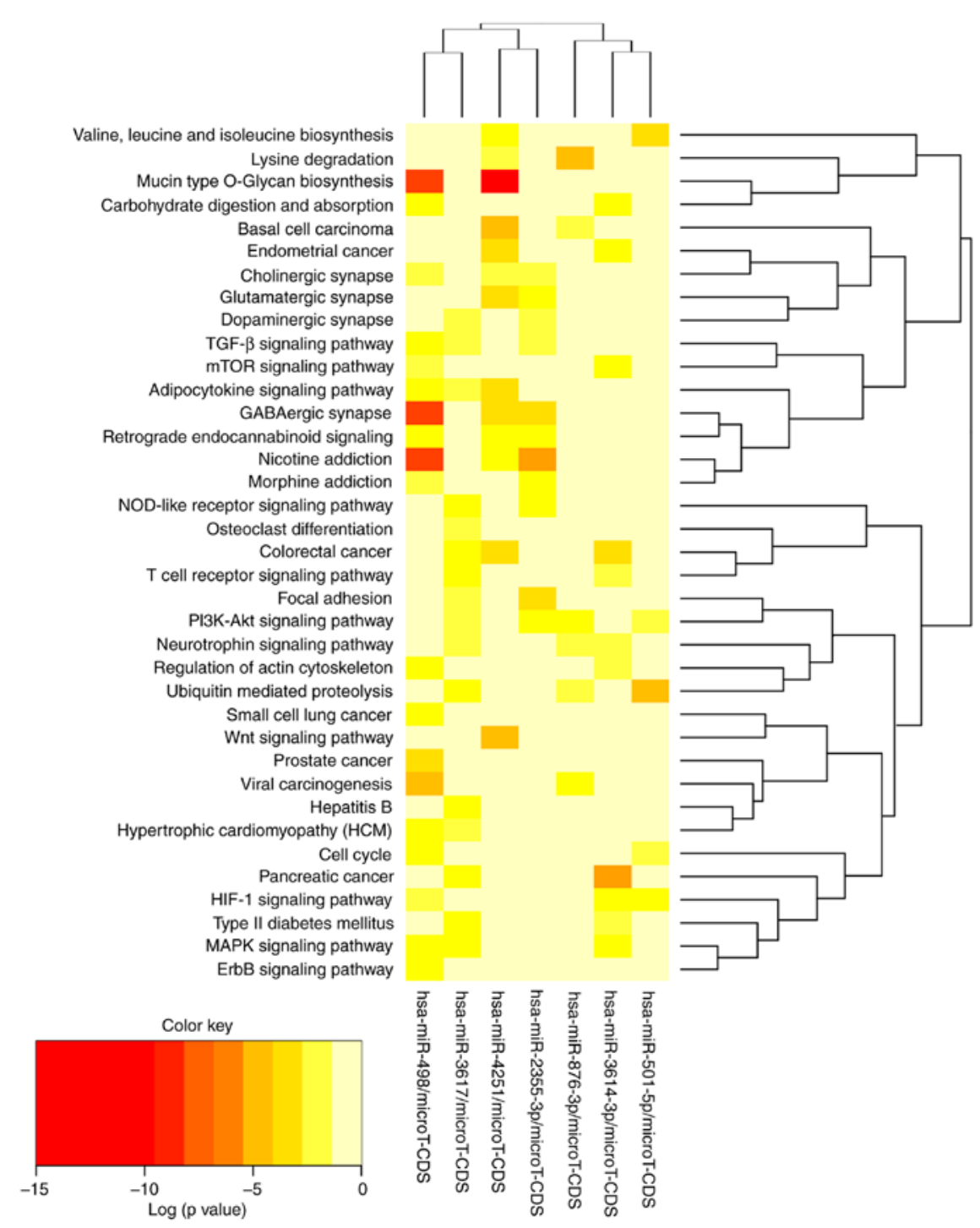

Figure 6. Prediction of circANKRD36/miR-mediated pathways. miR, microRNA; circANKRD36, circular RNA ankyrin repeat domain 36. 
associated with the T2DM pathway and mitogen-activated protein kinase (MAPK) pathway (Fig. 6). The MAPK signaling pathway can be activated by hyperglycemia, and is also involved in inflammation and immunization (26-28). These results indicated that circANKRD36 was associated with the inflammatory state in T2DM. However, further experiments are required to confirm the connection between circRNAs and miRNAs, other than prediction by bioinformatics software, including the use of fluorescence in situ hybridization assay, RNA immunoprecipitation and luciferase activity assays, and the transfection of a circRNA overexpression vector. In future investigations, experiments are to be continued to confirm the predicted connection between circANKRD36 and miRNAs, including hsa-miR-3617 and hsa-miR-3614-3p.

In conclusion, the present study demonstrated that circANKRD36 was expressed at high levels in leucocytes of patients with T2DM. Furthermore, the expression of circANKRD36 was associated with clinical parameters and inflammatory factors. circANKRD36 may not only be a potential biomarker, but may also be involved in pathogenesis. The underlying mechanism between circANKRD36 and chronic, low-grade inflammation in patients with $\mathrm{T} 2 \mathrm{DM}$ requires further investigation.

\section{Acknowledgements}

The authors would like to thank Dr Chuanbao Li (Beijing Hospital, China) for his help in sample collection.

\section{Availability of data and materials}

The datasets used and/or analyzed during the current study are available from the corresponding author on reasonable request.

\section{Funding}

The present study was supported by the Beijing Natural Science Foundation (grant no. 7172193) and the National Natural Science Foundation of China (grant no. 81571384).

\section{Authors' contributions}

YF and LZ designed the experiments, performed the experiments, analyzed the data, and wrote the manuscript; XW, FS and QP provided the patient samples, conceived the study and revised the manuscript; WL, JH, JZ and WH performed the experiments, analyzed the data and revised the manuscript; JJ and FX designed the experiments, provided vital reagents and revised the manuscript.

\section{Ethics approval and consent to participate}

The study was performed in accordance with the Declaration of Helsinki, and was approved by the Ethics Committee of Beijing Hospital (no. 2015BJYYEC-066-02). The participants provided written informed consent to participate in this study.

\section{Patient consent for publication}

Consent for publication was obtained from all participants.

\section{Competing interests}

The authors declare that they have no competing interests.

\section{References}

1. Shoelson SE, Lee J and Goldfine AB: Inflammation and insulin resistance. J Clin Invest 116: 1793-1801, 2006.

2. Seidell JC: Obesity, insulin resistance and diabetes-a worldwide epidemic. Br J Nutr 83 (Suppl 1): S5-S8, 2000.

3. Rahelić D: 7th edition of IDF diabetes atlas-call for immediate action. Lijec Vjesn 138: 57-58, 2016 (In Croatian).

4. Ma RC, Lin X and Jia W: Causes of type II diabetes in China. Lancet Diabetes Endocrinol 2: 980-991, 2014.

5. Chen L, Chen R, Wang H and Liang F: Mechanisms linking inflammation to insulin resistance. Int $\mathbf{J}$ Endocrinol 2015: 508409, 2015.

6. Memczak S, Jens M, Elefsinioti A, Torti F, Krueger J, Rybak A, Maier L, Mackowiak SD, Gregersen LH, Munschauer M, et al: Circular RNAs are a large class of animal RNAs with regulatory potency. Nature 495: 333-338, 2013.

7. Hansen TB, Jensen TI, Clausen BH, Bramsen JB, Finsen B, Damgaard CK and Kjems J: Natural RNA circles function as efficient microRNA sponges. Nature 495: 384-388, 2013.

8. Zhang Y, Zhang XO, Chen T, Xiang JF, Yin QF, Xing YH, Zhu S, Yang L and Chen LL: Circular intronic long noncoding RNAs. Mol Cell 51: 792-806, 2013.

9. Li F, Zhang L, Li W, Deng J, Zheng J, An M, Lu J and Zhou Y: Circular RNA ITCH has inhibitory effect on ESCC by suppressing the $\mathrm{Wnt} / \beta$-catenin pathway. Oncotarget 6 : 6001-6013, 2015 .

10. Jeck WR, Sorrentino JA, Wang K, Slevin MK, Burd CE, Liu J, Marzluff WF and Sharpless NE: Circular RNAs are abundant, conserved, and associated with ALU repeats. RNA 19: 141-157, 2013.

11. Memczak S, Papavasileiou P, Peters O and Rajewsky N: Identification and characterization of circular RNAs as a new class of putative biomarkers in human blood. PLoS One 10: e0141214, 2015.

12. Zhao Z, Li X, Jian D, Hao P, Rao L and Li M: Hsa_circ_0054633 in peripheral blood can be used as a diagnostic biomarker of pre-diabetes and type 2 diabetes mellitus. Acta Diabetol 54: 237-245, 2017.

13. Xu H, Guo S, Li W and Yu P: The circular RNA Cdr1 as, via miR-7 and its targets, regulates insulin transcription and secretion in islet cells. Sci Rep 5: 12453, 2015.

14. Livak KJ and Schmittgen TD: Analysis of relative gene expression data using real-time quantitative PCR and the 2(-Delta Delta C(T)) method. Methods 25: 402-408, 2001.

15. Huang HY, Chien CH, Jen KH and Huang HD: RegRNA: An integrated web server for identifying regulatory RNA motifs and elements. Nucleic Acids Res 34: W429-W434, 2006.

16. Vlachos IS, Zagganas K, Paraskevopoulou MD, Georgakilas G, Karagkouni D, Vergoulis T, Dalamagas T and Hatzigeorgiou AG: DIANA-miRPath v3.0: Deciphering microRNA function with experimental support. Nucleic Acids Res 43: W460-W466, 2015.

17. Salzman J, Chen RE, Olsen MN, Wang PL and Brown PO: Cell-type specific features of circular RNA expression. PLoS Genet 9: e1003777, 2013.

18. Keane KN, Cruzat VF, Carlessi R, de Bittencourt PI Jr and Newsholme P: Molecular events linking oxidative stress and inflammation to insulin resistance and $\beta$-cell dysfunction. Oxid Med Cell Longev 2015: 181643, 2015.

19. Hotamisligil GS, Shargill NS and Spiegelman BM: Adipose expression of tumor necrosis factor-alpha: Direct role in obesity-linked insulin resistance. Science 259: 87-91, 1993.

20. Aldhahi $\mathrm{W}$ and Hamdy O: Adipokines, inflammation, and the endothelium in diabetes. Curr Diab Rep 3: 293-298, 2003.

21. Pradhan AD, Manson JE, Rifai N, Buring JE and Ridker PM: C-reactive protein, interleukin 6 , and risk of developing type II diabetes mellitus. JAMA 286: 327-334, 2001.

22. Huang J, Yang Y, Hu R and Chen L: Anti-interleukin-1 therapy has mild hypoglycaemic effect in type II diabetes. Diabetes Obes Metab 20: 1024-1028, 2018. 
23. Maedler K, Sergeev P, Ris F, Oberholzer J, Joller-Jemelka HI, Spinas GA, Kaiser N, Halban PA and Donath MY: Glucose-induced $\beta$ cell production of IL-1 $\beta$ contributes to glucotoxicity in human pancreatic islets. J Clin Invest 110: 851-860, 2002.

24. Li Z, Huang C, Bao C, Chen L, Lin M, Wang X, Zhong G, Yu B, $\mathrm{Hu}$ W, Dai L, et al: Exon-intron circular RNAs regulate transcription in the nucleus. Nat Struct Mol Biol 22: 256-264, 2015.

25. Sengupta U,Ukil S,Dimitrova N and Agrawal S: Expression-based network biology identifies alteration in key regulatory pathways of type II diabetes and associated risk/complications. PLoS One 4: e8100, 2009.

26. Xia F, Wang C, Jin Y, Liu Q, Meng Q, Liu K and Sun H: Luteolin protects HUVECs from TNF- $\alpha$-induced oxidative stress and inflammation via its effects on the Nox4/ROS-NF- $\kappa$ B and MAPK pathways. J Atheroscler Thromb 21: 768-783, 2014.
27. Seo MJ, Lee YJ, Hwang JH, Kim KJ and Lee BY: The inhibitory effects of quercetin on obesity and obesity-induced inflammation by regulation of MAPK signaling. J Nutr Biochem 26: 1308-1316, 2015.

28. Adhikary L, Chow F, Nikolic-Paterson DJ, Stambe C, Dowling J, Atkins RC and Tesch GH: Abnormal p38 mitogen-activated protein kinase signalling in human and experimental diabetic nephropathy. Diabetologia 47: 1210-1222, 2004.

This work is licensed under a Creative Commons Attribution-NonCommercial-NoDerivatives 4.0 International (CC BY-NC-ND 4.0) License. 